therefore, that the authors failed to demonstrate larger ventricles in schizophrenic subjects, since their sample size (30 patients, 30 controls) gave them slightly less than a 50-50 chance of doing so.

The authors should be congratulated on a genuinely informative literature review, but it is a shame they felt unable to put their own prescriptions into practice. In illuminating the devil of the Type I error they have slipped into the deep blue sea of the Type II.

Institute of Psychiatry

De Crespigny Park

Denmark Hill

London SE5 8AF

\section{Schizophrenia: Recency Theory and Syphilis}

SIR: Hare (Journal, October 1988, 153, 521-531) argued that the historical origins of schizophrenia may have parallels with that of general paresis. He described the earlier global variation in the prevalence of paresis and the more recent increase in prevalence in non-Western countries, and judged from this that the changes in prevalence were real and probably attributable to changes in an environmental factor, i.e. the introduction of a new infectious agent.

The relative rarity of syphilis, and by extension of general paresis, in West Africa before 1950 may have been related to the endemicity of yaws; the rapid increase in the incidence of syphilis since then is thought to be related to the decrease in the prevalence of yaws following the WHO mass treatment campaign of the 1950s and 1960s (Perine, 1987).

Yaws is caused by Treponema pertenue, which is morphologically identical to Treponema pallidum, the cause of venereal syphilis. The increase in venereal syphilis since the mass treatment programmes may represent a decline in herd immunity to yaws, and thereby to syphilis. This thesis is not incompatible with the notion that venereal syphilis was a new disease. Venereal syphilis may well have derived from the endemic treponematoses (unitarian theory) which include pinta, yaws, bejel, and endemic syphilis (Csonka, 1987). However, the thesis suggests that rapid alterations in prevalence, especially in this case, may be as much to do with changes in herd immunity to a related pathogen as it is to do with introduction of a new pathogen.

I agree with Dr Hare that study of the historical origins of general paresis may throw some light on the schizophrenia debate and suggest that the curious relationship between rheumatoid arthritis and schizophrenia (if true) is reminiscent of that between yaws and venereal syphilis in the African population. Both rheumatoid arthritis and schizophrenia may be caused by related but different pathogens.

\section{John Conolly Hospital \\ Bristol Road \\ South Rednal \\ Birmingham B45 9BD}

FEMI OYEBODE

\section{References}

CsonkA, G. W. (1987) Syphilis. In Oxford Textbook of Medicine (2nd edn) (eds D. J. Weatherall, J. G. G. Ledingham \& D. A. Warrell). Oxford: Oxford University Press.

PerINE, P. C. (1987) Non-venereal treponematoses: yaws, endemic syphilis, and pinta. In Oxford Textbook of Medicine (2nd edn) (eds D. J. Weatherall, J. G. G. Ledingham \& D. A. Warrell). Oxford: Oxford University Press.

\section{Tactile Extinction Phenomenon in Schizophrenia}

SIR: With regard to Dr Cutting's comments (Journal, August 1988, 153, 281) about our interpretation of the tactile extinction phenomenon in his review of Etiopathogenetic Hypotheses of Schizophrenia: the impact of epidemiological, biochemical and neuromorfological studies, the direct relationship between QET and "a disorder of some sort in the right hemisphere" is so far to be univocally demonstrated. Taking the neurophysiological pathway of tactile sensitivity into account, there is clinical evidence that both right contralateral and left frontal cerebral lesions cause a left-extinction phenomenon (Schwartz et al, 1977).

Attempts to clarify which of these (left homolateral or right contralateral) is responsible for our results have not given consistent results. Even if a neuropsychological interpretation of the QET suggests that left-extinguishing patients performed worse in right-hemisphere tasks (Gambini et al, 1986) the interpretation of these results still are not definitive. In fact, QET can be considered to be similar to other dichotic tasks (e.g. dichotic listening and, in general, simultaneous stimulations of the left and right sensorial pathways). Therefore, as Schwartz et al $(1977,1979)$ pointed out, it is reasonable to suggest that when a verbal response is needed, as in the QET paradigm, the two sensorial tactile pathways are quite different; in fact, the left-side stimulus has a longer indirect pathway to CNS in comparison with the stimulus from the right hand, which arrives directly to the left hemisphere. The longer way for the left-side stimulus includes fibres crossing from the right to the left hemisphere mainly through the anterior part of the corpus callosum. Thus it might be reasonably 
hypothesised that the tactile extinction phenomenon in schizophrenia might also depend on transcallosal pathway deficits.

Psychiatric Branch

O. GAMBINI

L. BELLINI

S. SCARONE

Department of Biomedical and Technological

Sciences

University of Milan Medical School

San Raffaele Hospital

via Prinetti 29, 20127 Milano, Italy

\section{References}

Gambini, O., Cazzullo, C. L. \& Scarone, S. (1986) Interpretation of abnormal response to the QET in schizophrenia. Journal of Neurology, Neurosurgery and Psychiatry, 49, 997-1001.

SChwartz, A. S., MarChok, P. L. \& FlynN, R. E. (1977) A sensitive test for tactile extinction: results in patients with parietal and frontal lobe disease. Journal of Neurology. Neurosurgery and Psychiatry, 40, 228-233

,-- KRENIC, C. J., et al (1979) The asymmetric lateralization of tactile extinction in patients with unilateral cerebral dysfunction. Brain, 102, 669-684.

\section{Violent Incidents in Special Hospitals}

SIR: We read with interest the paper by Larkin et al (Journal, August 1988, 153, 226-231). Results from our own investigation of violent incidents over a 12 month period at another Special Hospital (Park Lane) agree in some respects, but differ substantially in others.

In common with Dr Larkin et al, we did not confirm previous findings of increased frequency of violence at mealtimes (Dietz \& Rada, 1982; Hodgkinson et al, 1985; Pearson et al, 1986). We also agree in that incidents generally took place in ward areas. Our study did not find a significantly greater number of incidents on Monday and Friday as did theirs. In our study, $59 \%$ of incidents occurred spontaneously, in contrast to their figure of $85 \%$. Our finding that staff and patients are equally likely to be targets of assault differs from their results, which indicate that nursing staff were three times more likely to be the victim of assault than the other patients. Park Lane has no female patients, and we are not able to comment on their finding that female patients were responsible for a disproportionate number of assaults.

Reasons for our differing findings may be found in the differing patient populations between the two hospitals - Rampton caters for all four of the categories of disorder in the Mental Health Act, whereas Park Lane only has mentally ill or psychopathic patients, of a level of intelligence within the normal range. Additionally, our study within Park Lane related almost exclusively to those patients with chronic mental illness.

Finally, our most striking finding was that of a marked seasonal variation in incidents, with significantly increased frequency of assault in the winter months. We postulate an important seasonal variation whereby increased population density in indoor areas, during cold and dark winter months, leads to increased levels of extrapunitive violence. Larkin et al report on incidents between May and September, the period when we recorded fewest incidents, and do not comment on a seasonal trend. In view of this, it may be that their calculation of an incident rate for the whole year may be seriously underestimated.

Park Lane Hospital

Park Lane

Maghull

Liverpool L31 $1 \mathrm{HW}$

J. B. COLDWELL

L. J. NAISMITH

\section{References}

DIETZ, P. E. \& RADA, R. T. (1982) Battery incidents and batterers in a maximum security hospital. Archives of General Psychiatry, 39, 31-34.

Hodgkinson, P. E., McIvor, L. \& Phillips, M. (1985) Patient assaults on staff in a psychiatric hospital: a two year retrospective study. Medicine, Science and the Law, 25, 228-294.

LARKin, E. P., MURTAGH, S. \& Jones, S. J. (1988) A preliminary study of violent incidents in a special hospital (Rampton). British Journal of Psychiatry, 153, 226-231.

Pearson, M., Wilmot, E. \& Padi, M. (1986) A study of violent behaviour among in-patients in a psychiatric hospital. British Journal of Psychiatry, 149, 232-235.

\section{Capgras' Syndrome}

SIR: Dr Anderson's intriguing case report (Journal, November 1988, 153, 694-699) adds more weight, if such were needed, to the notion that delusional misidentification syndromes can be said always to signal the presence of cerebral pathology. By my calculation, 21 of the 23 cases of Capgras' syndrome now reported with neuroimaging data have shown focal or diffuse abnormalities: an impressive score even allowing for publication bias. One small caveat, which Dr Anderson acknowledges, is that the case he describes is not classically a Capgras delusion. Previous authors have included the 'delusion of inanimate doubles' within that sublime group of neurological disorders called the reduplicative paramnesias (Weinstein, 1969). However, such fine distinctions are mainly in the eye of the beholder, be he psychiatrist or neurologist. The underlying mechanism offered by Dr Anderson, that of a lesion 